\title{
Effect of Stiffening System on Building Resistance to Earthquake Forces
}

\author{
Nabeel A. Jasim ${ }^{1}$, MohammedF.Ojaimi ${ }^{2}$ \\ ${ }^{1,2}$ (Civil Engineering Department, University of Basrah, Iraq)
}

\begin{abstract}
Multi-story steel buildings of various heights under the action of earthquake force are analyzed by using time-history analysis technique. The ground motion records of El Centro, California in 1940 are considered in this study. Different types of stiffening systems (bracing and shear walls) are used for the considered buildings. The main objective of this study is to evaluate the response of steel structures subjected to earthquake excitation and to investigate the effect of various stiffening systems in improving the response of these buildings. The finite element method of SAP 2000 V17program is used in the analysis. A static analysis is conducted to obtain an indication on the stiffness of the studied stiffening models in order to interpret the stiffness effect on the response of the structures under the seismic load. It is found that, the natural period of a structure is highly affected by the height of the structure and the used stiffeningsystem. It is inversely proportional with the stiffness and directly proportional with the height of the structure. It is concluded that the roof displacement andits maximum value at a specific momentdoes not give a clear indication for the behavior of building. Therefore the full time response of the building must be considered. Also it has been concluded that it is not necessarily when the stiffness of a building increases, the roof or any story displacement of the building decreases under earthquake load.
\end{abstract}

Keywords: Earthquake Force, Seismic Load, Bracing, Shear Wall, Time-History, stiffening, Steel Buildingsand Free Vibration.

\section{Introduction}

Earthquakes are one of the most devastating natural hazards that cause great loss of life and livelihood. An earthquake is one of the periodic adjustments that the earth makes in its evolution [1]. If the base of a structure is suddenly moved, as in a seismic event, the upper part of the structure will not respond instantaneously, but will lag because of the inertial resistance and flexibility of the structure [2]. The resulting stresses and distortions in the building are the same as if the base of the structure is to remain stationary while time-varying horizontal forces are applied to the upper part of the building[2].These forces, called inertia forces. In general, tall buildings respond to seismic motion differently than low-rise buildings. The magnitude of inertia forces induced in an earthquake depends on the building mass, ground acceleration, the nature of the foundation, and the dynamic characteristics of the structure [2]. The solution of a typical structural dynamic problem is considerably more complicated than its static counterpart. This is due to the addition of new forces of inertia and damping to the forces of elastic resistance of the system, and due to the time dependency of all of these forces. The dynamic problem from time dependency standpoint is not simply solving as the static problem only, but it is different in character [3].

The performance of buildings under earthquake load has been the subject of many studies over a number of years. Pundkar and Alandkar, 2013[4], modeled four high-rise steel buildings having nineteen stories and situated in zone III according to IS 1893:2002 [5]with different steel plate shear wall (SPSW) locations. The buildings were analyzed for same geometry and loading. The analysis of steel plate shear wall building was carried out by using the response spectrum analysis of SAP2000 V15Software. The results revealed that due to presence of SPSW the total weight of steel in building decreased. Also it is observed that placing the shear wall away from the center of gravity resulted in the increase in lateral deflection. Verma, et. al, 2014[6], investigated the structural behavior of the buildings with shear walls at different locations. Tenstories and fifteen-stories reinforced concrete frames situated in seismic zone V, as per IS 1893:2002, have been analyzed by using the seismic coefficient method of STAAD PROsoftware. It was found that with the incorporation of shear walls story drift and average displacement decrease considerably. Dhiman, et. al, 2015[7], investigated the seismic performance of a fourteen story steel building considering different bracing patterns in seismic zone IV as per IS 1893:2002. STAAD V8i software was used. It has been concluded that the maximum reduction in the lateral displacement occurred after the use of cross bracing system. Chadhar and Sharma, 2015[8], used Staad ProV8i program and studied the response of the reinforced concrete (RC) moment resisting bare frame and RC moment resisting frame with steel bracing system or with shear wall system. Three different heights of building as low rise, medium rise and high rise were taken. It was found that RC moment resisting building frame with different types of bracing system and different configurations 
of shear walls have better resistance against earthquake forces. Also it was concluded that the steel bracing system provides better performance than shear wall system for various heights of building. In this study timehistory analysis technique is used to analyze different building models by using SAP2000 V17 software. In time-history analysis method, the mathematical model is subjected to full range of accelerations for the entire duration of earthquake by using earthquake records that represent the expected earthquake at the base of the structure.

\section{Structuralconfiguration}

\section{Structural Modeling}

The structural frame model of Ref. [9] is used in this study. The Indian standard IS 800:2007[10] is considered in the design of the steel structure. IS 875-1 (1987) [11] and IS 875-2 (1987) [12] are used to evaluate the applied dead and live load, respectively. The studied models are analyzed by time-history analysis technique.In this study eleven buildings are considered.All buildings have similar plan dimensions but differ in the number of stories. The number of stories included in the investigation are $(\mathrm{G}+4,6,8,10,12$, 14, 16, 18, 20, 30 and 40). The considered steel frames are provided with different stiffening systems. As defined in Table (1), eleven models are proposed for each building of specific number of stories. The first model is a rigid bare frame, followed by six rigid steel frames with different patterns of concentric steel bracing systems. The bracing members are provided at the peripheral edges of the building. Other three models of rigid steel frames with different positions of reinforced concrete shear wall (250 mm thick) are used. The last considered model is a hybrid frame which includes both the shear wall and bracing systems.

The plan of the steel framed building and the description of different models for ten stories $(\mathrm{G}+10)$ building are shown in Fig.(1). Similar configurations are used for the other studied buildings with different number of stories. The dimensions in plan of the studied buildings are $36 \mathrm{~m}$ (in X direction) and $30 \mathrm{~m}$ (in $\mathrm{Y}$ direction). The bays are $(6 \mathrm{~m} * 6 \mathrm{~m})$ center to center in both directions with six bays in $\mathrm{X}$ direction and five bays in $\mathrm{Y}$ direction. Each building essentially consists of deck concrete slab (150 mm thick) resting on the steel beams. This slab and beam structural system is supported on the steel columns. Typical floor to floor heights measured from centers of beams, including the ground floor, are $3 \mathrm{~m}$. The column bases are modeled as fixed at the ground level.

Table (1) Description of stiffening systems for the considered models.

\begin{tabular}{|r|l|l|}
\hline \multicolumn{1}{|l|}{ Model No. } & Improvingsystem & Description ofimproving system \\
\hline$(1)$ & Withoutimproving & ---- \\
\hline$(2)$ & Bracing & X bracing at the corners \\
\hline$(3)$ & Bracing & diagonal bracing \\
\hline$(4)$ & Bracing & double X bracing \\
\hline$(5)$ & Bracing & inverse V bracing \\
\hline$(6)$ & Bracing & V bracing \\
\hline$(7)$ & & full X bracing \\
\hline$(8)$ & Shear wall & shear wallsat corners \\
\hline$(9)$ & Shear wall & shear walls at the coreof the building \\
\hline$(10)$ & Shear wall & shear walls and the peripheraledges of the building \\
\hline$(11)$ & Bracing andShear wall & \\
\hline
\end{tabular}

The mass of each element is assumed to be concentrated at the nodes. The distribution of element mass is equally divided between nodes. The mass of the structure includes the applied dead load, including the self-weight, and live load. The mass is equal to the weight defined by the dead and live load divided by the gravitational acceleration $(\mathrm{g})$. All the dead load and only 25 percent of the live load is considered as per IS 1893-1 (2002) [5].

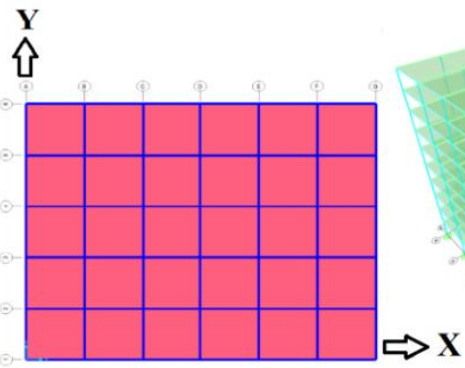

Plan diagram

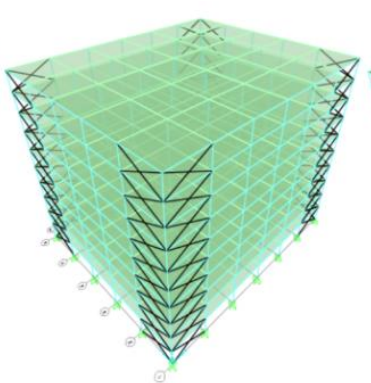

Model (1) Model (2) Model (3)

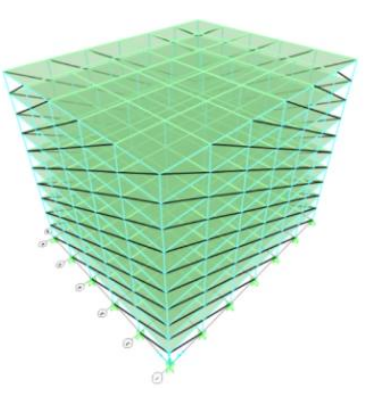



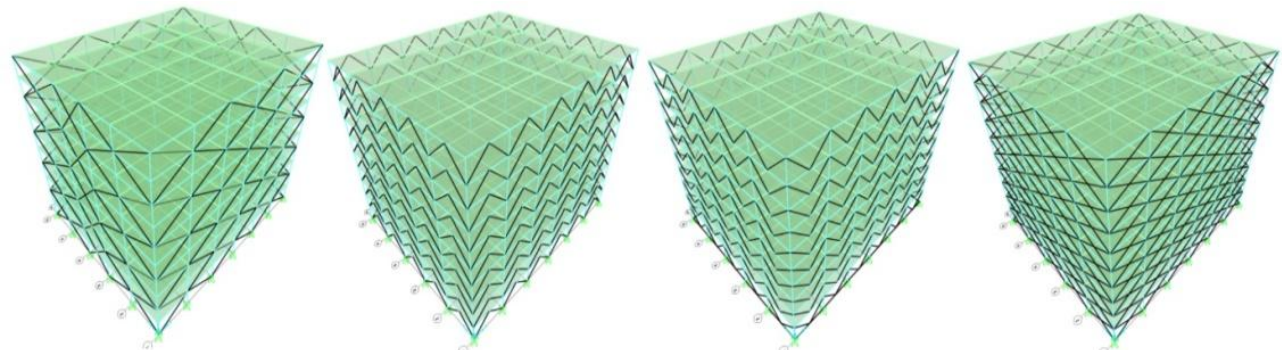

Model (4) Model (5)Model (6)Model (7)
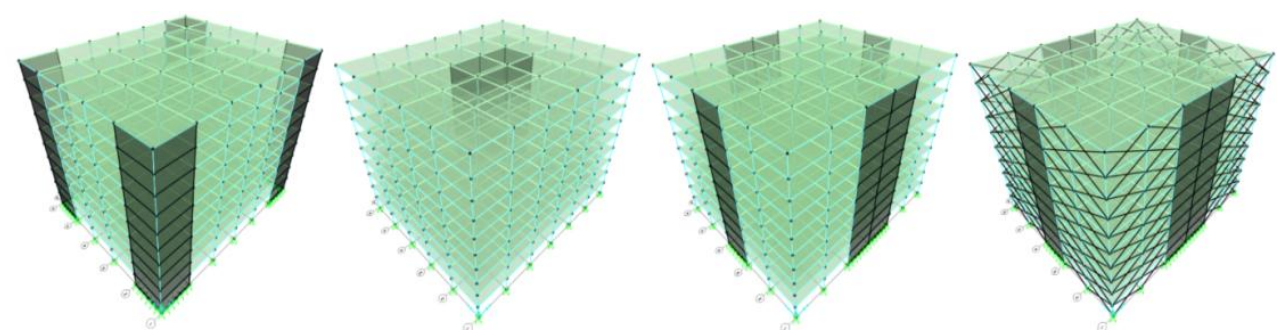

Model (8) Model (9) Model (10)Model (11)

Figure (1) Plan diagram and steel framed models of $(\mathrm{G}+10)$ stories building.

\section{Member sizes}

The section sizes of the used beams, columns and bracings are shown in Table (2).All the beams and bracing members in the studied buildings are I- beam section ISMB 550 for beams and BR $230 * 230 * 40$ angle section for bracings. Sections of columns are reduced from bottom to top which is same for every ten stories. The column sizes along the height of the buildings with different number of stories are summarized in Table (3).

Table (2) Size of beams, columns and bracings.

\begin{tabular}{|c|c|c|c|c|}
\hline \multirow{2}{*}{ Sections } & \multicolumn{4}{|c|}{ All dimensions in $\mathrm{mm}$} \\
\hline & Depth & $\begin{array}{c}\text { Flange } \\
\text { width }\end{array}$ & $\begin{array}{c}\text { Flange } \\
\text { thickness }\end{array}$ & $\begin{array}{c}\text { Web } \\
\text { thickness }\end{array}$ \\
\hline $\begin{array}{c}\text { I-Beam } \\
\text { ISMB } 550\end{array}$ & 550 & 190 & 19.3 & 11.2 \\
\hline $\begin{array}{l}\text { Column } \\
\text { CI } 1200 \\
\end{array}$ & 1200 & 850 & 40 & 40 \\
\hline $\begin{array}{l}\text { Column } \\
\text { CI } 1000\end{array}$ & 1000 & 600 & 35 & 35 \\
\hline $\begin{array}{l}\text { Column } \\
\text { CI } 900\end{array}$ & 900 & 500 & 35 & 35 \\
\hline $\begin{array}{l}\text { Column } \\
\text { CI } 800 \\
\end{array}$ & 800 & 450 & 30 & 30 \\
\hline $\begin{array}{l}\text { Bracingangle } \\
\text { BR } 230^{*} 230^{*} 40\end{array}$ & \multicolumn{2}{|c|}{ Leg length $=230$} & \multicolumn{2}{|c|}{ Thickness $=40$} \\
\hline
\end{tabular}

Table (3) Columns sizes along the height of the studied buildings

\begin{tabular}{|c|c|c|c|c|}
\hline $\begin{array}{l}\text { No. of } \\
\text { stories }\end{array}$ & $\begin{array}{c}\text { Stories } \\
\text { having } \\
\text { CI 1200 } \\
\text { Column } \\
\text { Section }\end{array}$ & $\begin{array}{c}\text { Stories } \\
\text { having } \\
\text { CI 1000 } \\
\text { Column } \\
\text { Section }\end{array}$ & $\begin{array}{c}\text { Stories } \\
\text { having } \\
\text { CI 900 } \\
\text { Column } \\
\text { Section }\end{array}$ & $\begin{array}{c}\text { Stories } \\
\text { having } \\
\text { CI 800 } \\
\text { Column } \\
\text { Section }\end{array}$ \\
\hline$(\mathrm{G}+4)$ & ----- & ----- & ----- & $1-4$ \\
\hline$(\mathrm{G}+6)$ & ----- & ----- & ----- & $1-6$ \\
\hline$(\mathrm{G}+8)$ & ----- & ----- & ----- & $1-8$ \\
\hline$(\mathrm{G}+10)$ & ----- & ----- & ----- & $1-10$ \\
\hline$(\mathrm{G}+12)$ & ----- & ----- & $1-10$ & $11-12$ \\
\hline$(\mathrm{G}+14)$ & ----- & ----- & $1-10$ & $11-14$ \\
\hline$(\mathrm{G}+16)$ & ----- & ----- & $1-10$ & $11-16$ \\
\hline$(\mathrm{G}+18)$ & ----- & ----- & $1-10$ & $11-18$ \\
\hline$(\mathrm{G}+20)$ & ----- & ----- & $1-10$ & $11-20$ \\
\hline$(\mathrm{G}+30)$ & ----- & $1-10$ & $11-20$ & $21-30$ \\
\hline$(\mathrm{G}+40)$ & $1-10$ & $11-20$ & $21-30$ & $31-40$ \\
\hline
\end{tabular}




\section{Applied loads}

The steel buildings are analyzed for dead, live, and earthquake loads. (a) Dead load: In addition to the self -weight, the floor dead load is taken as $\left(2 \mathrm{kN} / \mathrm{m}^{2}\right)$ and the roof dead load as $\left(4 \mathrm{kN} / \mathrm{m}^{2}\right)$. (b) Live load: The floor live load is taken as $\left(3 \mathrm{kN} / \mathrm{m}^{2}\right)$ and the roof live load as $\left(1.5 \mathrm{kN} / \mathrm{m}^{2}\right)$. According to the IS $1893-1$ (2002) [5], in the seismic analysis, all dead load and only 25 percent of the live load is to be considered when the imposed uniformly distributed floor load is less than or equal to $\left(3 \mathrm{kN} / \mathrm{m}^{2}\right)$. (c)earthquake functional load: All the studied models are subjected to the north - south component of the ground motion recorded in $\mathrm{X}$ direction at the site in El Centro, California in 1940. The acceleration values are applied to the base of the buildings during 50 seconds for each 0.02 second time intervals.

\section{Material properties}

Steel is used in modeling of beams, columns and bracing members. Reinforced concrete is used in modeling of the deck slabs and shear walls. Damping ratio of $(\zeta=0.02)$ is assumed. The material properties for the used steel and reinforced concrete are presented in Tables (4) and (5), respectively.

Table (4) Steel properties. Table (5) Reinforced concrete properties.

\begin{tabular}{|c|c|c|c|c|c|c|c|}
\hline Item & Description & Unit & Value & Item & Description & Unit & Value \\
\hline $\mathrm{f}_{\mathrm{y}}$ & Minimum yield stress & $\mathrm{N} / \mathrm{mm}^{2}$ & 250 & $f_{c}^{\prime}$ & Cylinder compression strength & $\mathrm{N} / \mathrm{mm}^{2}$ & 27.5 \\
\hline$F_{u}$ & Ultimate tensile stress & $\mathrm{N} / \mathrm{mm}^{2}$ & 410 & $f_{y}$ & $\begin{array}{c}\text { Yield stress ofsteel } \\
\text { reinforcement }\end{array}$ & $\mathrm{N} / \mathrm{mm}^{2}$ & 420 \\
\hline $\mathrm{E}_{5}$ & Modulus of elasticity & $\mathrm{N} / \mathrm{mm}^{2}$ & 210000 & $E_{c}$ & $\begin{array}{l}\text { Modulus of elasticity } \\
\text { of concrete }\end{array}$ & $\mathrm{N} / \mathrm{mm}^{2}$ & 25000 \\
\hline$\rho_{5}$ & Density & $\mathrm{kN} / \mathrm{m}^{3}$ & 77 & $\rho_{c}$ & Density & $\mathrm{kN} / \mathrm{m}^{3}$ & 24 \\
\hline$v_{5}$ & Poisson's ratio & --- & 0.3 & $v_{\mathrm{c}}$ & Poisson's ratio & --- & 0.2 \\
\hline
\end{tabular}

\section{Finite Element Modeling}

The finite element method is one of the most powerful methods for solving partial differential equations, particularly when these equations are applied over complex shapes with complex boundary conditions [13].

\section{Types of used elements}

In this study the finite elements analysis program SAP2000 is used to formulate and analyze the whole system.This program contains several types of elements which can be used toformulate the structural problems. For the superstructure, two different types of elements are used.The first type is SHELL element (AREA element), which is a four-node quadrilateral shellelement with both bending and membrane capabilities as shown in Fig. (2).This element is used to simulate the slabs and walls of the structure. The element has six degrees of freedom at each node: translations in thex, $\mathrm{y}$, and $\mathrm{z}$ directions and rotations about the $\mathrm{x}, \mathrm{y}$, and $\mathrm{z}$-axes.

The second type of element is the BEAM element (FRAME element) with axial force, torsion, and bending capabilities.This element is used to represent beams, columns and bracing of the structure.A Frame element is modeled as a straight line connecting two points. The element has six degrees of freedom at each node: translations in the $\mathrm{x}, \mathrm{y}$, and $\mathrm{z}$ directions and rotations about the $\mathrm{x}, \mathrm{y}$, and $\mathrm{z}$ axes as shown in Fig. (3).
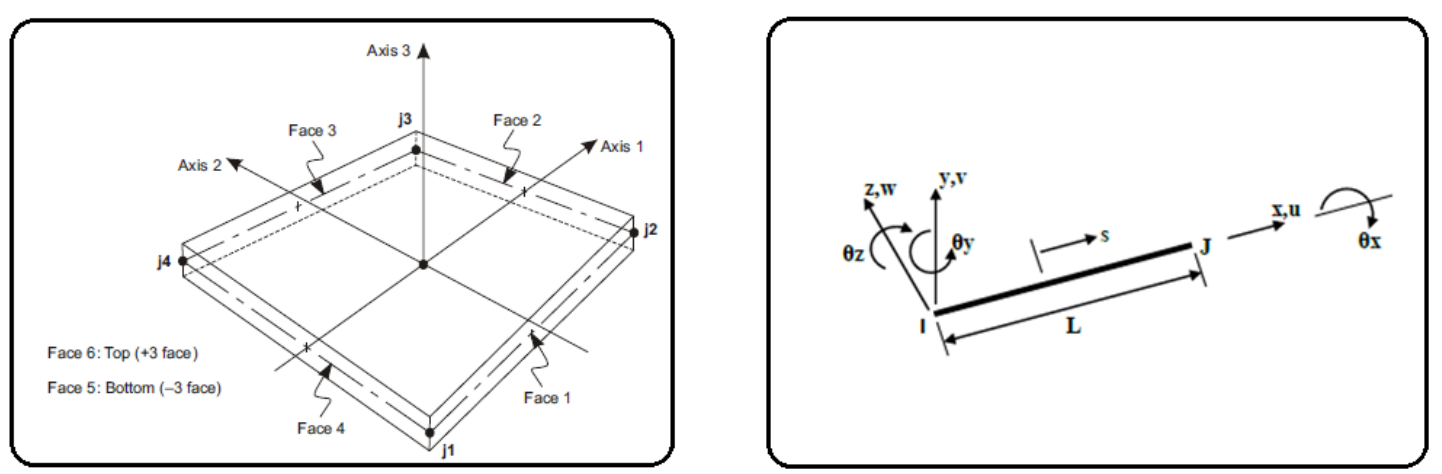

Figure (2) Four node quadrilateral shell element[14] Figure (3) Frame element[14]

\section{Mesh Size}

Eight models with different mesh sizes are examined to select a suitable mesh size which gives results with acceptable accuracy along with relatively less time to complete the analysis process. The main 
parameters considered for this purpose are the maximum roof displacement in $\mathrm{X}$ direction, maximum base shear in $\mathrm{X}$ direction and the fundamental natural period. The ten stories $(\mathrm{G}+10)$ rigid steel frame is analyzed and the examined mesh sizes are $0.25 \mathrm{~m}, 0.5 \mathrm{~m}, 0.75 \mathrm{~m}, 1 \mathrm{~m}, 1.25 \mathrm{~m}, 1.5 \mathrm{~m}, 2 \mathrm{~m}$ and $3 \mathrm{~m}$. The results of the study are presented in Table (6). It has been found that the model with mesh size of $1 \mathrm{~m}$ shows acceptable results for all studied parameters. Therefore, mesh size of $1 \mathrm{~m}$ is used throughout this study.

Table (6) Results of models with different mesh sizesfor $(\mathrm{G}+10)$ stories rigid steel frame.

\begin{tabular}{|c|c|c|c|c|c|c|c|}
\hline \multirow{3}{*}{$\begin{array}{r}\text { Model } \\
\text { No. }\end{array}$} & \multirow{3}{*}{$\begin{array}{r}\text { Mesh Size } \\
\left(\mathrm{m}^{*} \mathrm{~m}\right)\end{array}$} & \multirow{3}{*}{$\begin{array}{lr} & \{1\} \\
\text { Max. } & \text { Roof } \\
\text { displacement in } \\
\text { X } & \text { direction } \\
& (\mathrm{mm})\end{array}$} & \multirow{3}{*}{$\begin{array}{r}\{2\} \\
\text { Max. } \\
\text { Base shear in X } \\
\text { direction }(\mathrm{kN})\end{array}$} & \multirow{3}{*}{$\begin{array}{l}\{3\} \\
\text { Fundamental time } \\
\text { period of first mode } \\
(\mathrm{sec})\end{array}$} & \multicolumn{3}{|r|}{ Ratio* } \\
\hline & & & & & & & \\
\hline & & & & & $\{1\}$ & $\{2\}$ & $\{3\}$ \\
\hline (1) & $0.25 * 0.25$ & 219.3 & 36160 & 1.44969 & 1 & 1 & 1 \\
\hline$(2)$ & $0.50 * 0.50$ & 217.5 & 35190 & 1.45396 & 0.99 & 0.97 & 0.997 \\
\hline (3) & $0.75 * 0.75$ & 217.0 & 34700 & 1.45592 & 0.99 & 0.96 & 0.996 \\
\hline (4) & $1.00 * 1.00$ & 216.6 & 34550 & 1.45720 & 0.99 & 0.96 & 0.995 \\
\hline$(5)$ & $1.25 * 1.25$ & 216.4 & 34450 & 1.45796 & 0.99 & 0.95 & 0.994 \\
\hline (6) & $1.50 * 1.50$ & 216.0 & 34330 & 1.45899 & 0.99 & 0.95 & 0.994 \\
\hline$(7)$ & $2.00 * 2.00$ & 215.9 & 34280 & 1.45941 & 0.98 & 0.95 & 0.993 \\
\hline$(8)$ & $3.00 * 3.00$ & 214.9 & 33920 & 1.46248 & 0.98 & 0.94 & 0.991 \\
\hline
\end{tabular}

(*) The ratio is relative to result of $0.25 \mathrm{~m}$ mesh size.

\section{Results}

The response of the studied structures is investigated through several parameters such as the story displacement, story drift, maximum roof displacement, average roof displacement, base shear and fundamental natural period. The results of average roof displacement $(\boldsymbol{\Delta})$, maximum roof displacement $(\boldsymbol{\delta})$, time of maximum roof displacement $(\mathbf{T})$, maximum base shear $(\mathbf{V})$ and the fundamental natural period $\left(\mathbf{T}_{\mathbf{n}}\right)$ for all considered buildings with different heights and different stiffening systems are presented in Table (7).The average roof displacement represents the average value of the 2500 results records, for each $0.02 \mathrm{sec}$, during $50 \mathrm{sec}$ of earthquake action.

Table (7) Results (avg. roof displacement, max. roof displacement, time of max. roof displacement, max. base shear and the fundamental natural time period) of all studied buildings

(a) $(\mathrm{G}+4) \mathrm{St}$.

\begin{tabular}{|c|c|c|c|c|c|}
\hline $\begin{array}{c}\text { Model } \\
\text { No. }\end{array}$ & $\begin{array}{c}(\boldsymbol{\Delta}) \\
\mathbf{m m}\end{array}$ & $\begin{array}{c}(\boldsymbol{\delta}) \\
\mathrm{mm}\end{array}$ & $\begin{array}{c}(\mathbf{T}) \\
\text { sec }\end{array}$ & $\begin{array}{c}(\mathbf{V}) \\
\mathrm{kN}\end{array}$ & $\begin{array}{c}\left(\mathbf{T}_{\mathbf{n}}\right) \\
\text { sec }\end{array}$ \\
\hline$(1)$ & 5.8 & 46.2 & 5.02 & 24110 & 0.60180 \\
\hline$(2)$ & 3.0 & 23.6 & 2.56 & 30120 & 0.30621 \\
\hline$(3)$ & 1.4 & 11.7 & 3.24 & 22550 & 0.26236 \\
\hline$(4)$ & 1.4 & 11.3 & 3.22 & 25280 & 0.23330 \\
\hline$(5)$ & 0.9 & 8.1 & 4.94 & 26370 & 0.20377 \\
\hline$(6)$ & 0.7 & 8.2 & 2.26 & 24970 & 0.21720 \\
\hline$(7)$ & 0.7 & 6.1 & 5.62 & 23010 & 0.19194 \\
\hline$(8)$ & 0.7 & 7.8 & 4.94 & 25060 & 0.18903 \\
\hline$(9)$ & 0.5 & 6.7 & 2.64 & 29990 & 0.25411 \\
\hline$(10)$ & 0.6 & 6.1 & 3.94 & 23040 & 0.25970 \\
\hline$(11)$ & 0.3 & 2.9 & 2.6 & 20840 & 0.16208 \\
\hline
\end{tabular}

(c) $(\mathrm{G}+8) \mathrm{St}$.

\begin{tabular}{|c|c|c|c|c|c|}
\hline $\begin{array}{c}\text { Model } \\
\text { No. }\end{array}$ & $\begin{array}{c}(\boldsymbol{\Delta}) \\
\mathbf{m m}\end{array}$ & $\begin{array}{c}(\boldsymbol{\delta}) \\
\mathbf{m m}\end{array}$ & $\begin{array}{c}(\mathbf{T}) \\
\text { sec }\end{array}$ & $\begin{array}{c}(\mathbf{V}) \\
\mathrm{kN}\end{array}$ & $\begin{array}{c}\left(\mathbf{T}_{\mathbf{n}}\right) \\
\text { sec }\end{array}$ \\
\hline$(1)$ & 32.4 & 199.4 & 5.80 & 40200 & 1.17100 \\
\hline$(2)$ & 15.2 & 116.0 & 2.22 & 49650 & 0.66474 \\
\hline$(3)$ & 7.3 & 41.6 & 3.56 & 40050 & 0.49977 \\
\hline$(4)$ & 6.3 & 40.3 & 5.04 & 47040 & 0.44030 \\
\hline$(5)$ & 3.2 & 29.2 & 2.66 & 41740 & 0.38718 \\
\hline$(6)$ & 3.8 & 27.8 & 2.66 & 37040 & 0.40606 \\
\hline$(7)$ & 3.5 & 32.4 & 4.50 & 56600 & 0.36094 \\
\hline$(8)$ & 7.6 & 47.8 & 3.58 & 43390 & 0.45345 \\
\hline$(9)$ & 3.9 & 35.5 & 4.5 & 53340 & 0.48673 \\
\hline$(10)$ & 4.9 & 42.4 & 4.74 & 45990 & 0.64670 \\
\hline$(11)$ & 2.2 & 20.1 & 2.52 & 59560 & 0.31593 \\
\hline
\end{tabular}

(b) $(\mathrm{G}+6) \mathrm{St}$.

\begin{tabular}{|c|c|c|c|c|c|}
\hline $\begin{array}{c}\text { Model } \\
\text { No. }\end{array}$ & $\begin{array}{c}(\boldsymbol{\Delta}) \\
\mathbf{m m}\end{array}$ & $\begin{array}{c}(\boldsymbol{\delta}) \\
\mathrm{mm}\end{array}$ & $\begin{array}{c}(\mathbf{T}) \\
\mathrm{sec}\end{array}$ & $\begin{array}{c}(\mathbf{V}) \\
\mathrm{kN}\end{array}$ & $\begin{array}{c}\left(\mathbf{T}_{\mathbf{n}}\right) \\
\text { sec }\end{array}$ \\
\hline$(1)$ & 13.0 & 115.5 & 2.22 & 33030 & 0.88605 \\
\hline$(2)$ & 7.4 & 42.0 & 3.56 & 31200 & 0.47390 \\
\hline$(3)$ & 3.6 & 32.7 & 4.52 & 41580 & 0.37890 \\
\hline$(4)$ & 3.2 & 23.8 & 2.60 & 34070 & 0.33423 \\
\hline$(5)$ & 2.6 & 23.4 & 2.54 & 48190 & 0.29302 \\
\hline$(6)$ & 3.2 & 24.1 & 2.56 & 46700 & 0.30917 \\
\hline$(7)$ & 1.9 & 15.9 & 2.52 & 38690 & 0.27375 \\
\hline$(8)$ & 2.8 & 21.7 & 2.58 & 31560 & 0.30582 \\
\hline$(9)$ & 1.6 & 11.9 & 3.04 & 30100 & 0.36876 \\
\hline$(10)$ & 2.4 & 22.7 & 2.54 & 43560 & 0.43927 \\
\hline$(11)$ & 0.7 & 8.6 & 2.26 & 37800 & 0.23554 \\
\hline
\end{tabular}

(d) $(\mathrm{G}+10) \mathrm{St}$.

\begin{tabular}{|c|c|c|c|c|c|}
\hline $\begin{array}{c}\text { Model } \\
\text { No. }\end{array}$ & $\begin{array}{c}(\boldsymbol{\Delta}) \\
\mathrm{mm}\end{array}$ & $\begin{array}{c}(\boldsymbol{\delta}) \\
\mathrm{mm}\end{array}$ & $\begin{array}{c}(\mathbf{T}) \\
\mathrm{sec}\end{array}$ & $\begin{array}{c}(\mathbf{V}) \\
\mathrm{kN}\end{array}$ & $\begin{array}{c}\left(\mathbf{T}_{\mathbf{n}}\right) \\
\mathrm{sec}\end{array}$ \\
\hline$(1)$ & 42.6 & 216.6 & 4.50 & 34550 & 1.45720 \\
\hline$(2)$ & 20.4 & 128.5 & 2.72 & 47630 & 0.87354 \\
\hline$(3)$ & 14.0 & 100.2 & 5.14 & 78580 & 0.62482 \\
\hline$(4)$ & 11.7 & 82.0 & 2.38 & 68530 & 0.55125 \\
\hline$(5)$ & 7.3 & 44.0 & 3.58 & 53420 & 0.48613 \\
\hline$(6)$ & 8.2 & 54.5 & 5.00 & 62820 & 0.50790 \\
\hline$(7)$ & 6.0 & 44.6 & 5.02 & 65130 & 0.45332 \\
\hline$(8)$ & 16.1 & 120.5 & 2.22 & 65520 & 0.62642 \\
\hline$(9)$ & 7.8 & 49.1 & 3.58 & 48980 & 0.65892 \\
\hline$(10)$ & 12.2 & 87.4 & 2.38 & 66770 & 0.87004 \\
\hline$(11)$ & 3.6 & 32.3 & 4.50 & 69350 & 0.40173 \\
\hline
\end{tabular}


Table (7), Continued.

(e) $(\mathrm{G}+12) \mathrm{St}$.

\begin{tabular}{|c|c|c|c|c|c|}
\hline $\begin{array}{c}\text { Model } \\
\text { No. }\end{array}$ & $\begin{array}{c}(\boldsymbol{(}) \\
\mathbf{m m}\end{array}$ & $\begin{array}{c}(\boldsymbol{\delta}) \\
\mathbf{m m}\end{array}$ & $\begin{array}{c}(\mathbf{T}) \\
\text { sec }\end{array}$ & $\begin{array}{c}(\mathbf{V}) \\
\mathrm{kN}\end{array}$ & $\begin{array}{c}\left(\mathbf{T}_{\mathbf{n}}\right) \\
\text { sec }\end{array}$ \\
\hline$(1)$ & 49.3 & 164.1 & 6.72 & 29330 & 1.69764 \\
\hline$(2)$ & 30.6 & 226.9 & 4.72 & 54780 & 1.06388 \\
\hline$(3)$ & 14.6 & 113.2 & 2.24 & 68500 & 0.74426 \\
\hline$(4)$ & 12.9 & 113.1 & 2.22 & 77310 & 0.65807 \\
\hline$(5)$ & 12.6 & 84.6 & 2.38 & 81370 & 0.58254 \\
\hline$(6)$ & 13.7 & 90.7 & 2.40 & 85750 & 0.60557 \\
\hline$(7)$ & 12.7 & 86.1 & 5.12 & 92510 & 0.54334 \\
\hline$(8)$ & 20.4 & 130.6 & 2.70 & 65150 & 0.80055 \\
\hline$(9)$ & 16.9 & 133.0 & 5.18 & 104400 & 0.83635 \\
\hline$(10)$ & 18.2 & 126.8 & 2.24 & 63230 & 1.07789 \\
\hline$(11)$ & 5.8 & 46.3 & 4.76 & 78900 & 0.48463 \\
\hline
\end{tabular}

(g) $(\mathrm{G}+16) \mathrm{St}$.

\begin{tabular}{|c|c|c|c|c|c|}
\hline $\begin{array}{c}\text { Model } \\
\text { No. }\end{array}$ & $\begin{array}{c}(\mathbf{\Delta}) \\
\mathbf{m m}\end{array}$ & $\begin{array}{c}(\boldsymbol{\delta}) \\
\mathbf{m m}\end{array}$ & $\begin{array}{c}(\mathbf{T}) \\
\text { sec }\end{array}$ & $\begin{array}{c}(\mathbf{V}) \\
\mathrm{kN}\end{array}$ & $\begin{array}{c}\left(\mathbf{T}_{\mathbf{n}}\right) \\
\text { sec }\end{array}$ \\
\hline$(1)$ & 49.8 & 237.0 & 8.82 & 26350 & 2.20250 \\
\hline$(2)$ & 45.3 & 152.6 & 6.78 & 35610 & 1.49439 \\
\hline$(3)$ & 42.4 & 204.8 & 5.84 & 90180 & 1.00156 \\
\hline$(4)$ & 25.1 & 164.3 & 5.76 & 84890 & 0.89133 \\
\hline$(5)$ & 27.9 & 154.1 & 12.62 & 100300 & 0.79385 \\
\hline$(6)$ & 21.8 & 124.0 & 2.66 & 75240 & 0.82190 \\
\hline$(7)$ & 19.9 & 117.5 & 2.24 & 86200 & 0.74311 \\
\hline$(8)$ & 40.6 & 221.0 & 4.52 & 53640 & 1.20061 \\
\hline$(9)$ & 30.0 & 207.4 & 5.78 & 81500 & 1.23286 \\
\hline$(10)$ & 37.2 & 243.3 & 4.76 & 78340 & 1.53412 \\
\hline$(11)$ & 15.9 & 115.0 & 5.14 & 133400 & 0.66916 \\
\hline
\end{tabular}

(i) $(\mathrm{G}+20) \mathrm{St}$.

\begin{tabular}{|c|c|c|c|c|c|}
\hline $\begin{array}{c}\text { Moded } \\
\text { No. }\end{array}$ & $\begin{array}{c}(\boldsymbol{\Delta}) \\
\mathbf{m m}\end{array}$ & $\begin{array}{c}(\mathbf{\delta}) \\
\mathbf{m m}\end{array}$ & $\begin{array}{c}(\mathbf{T}) \\
\text { sec }\end{array}$ & $\begin{array}{c}(\mathbf{V}) \\
\mathbf{k N}\end{array}$ & $\begin{array}{c}\left(\mathbf{T}_{\mathbf{n}}\right) \\
\text { sec }\end{array}$ \\
\hline$(1)$ & 81.6 & 335.9 & 6.66 & 38740 & 2.72803 \\
\hline$(2)$ & 60.4 & 267.9 & 8.90 & 37640 & 1.96049 \\
\hline$(3)$ & 36.0 & 204.6 & 4.52 & 55500 & 1.27860 \\
\hline$(4)$ & 34.4 & 227.7 & 4.46 & 72300 & 1.14582 \\
\hline$(5)$ & 30.6 & 210.8 & 5.94 & 92470 & 1.02719 \\
\hline$(6)$ & 27.0 & 218.8 & 4.72 & 101200 & 1.06113 \\
\hline$(7)$ & 40.0 & 213.2 & 5.82 & 115400 & 0.96669 \\
\hline$(8)$ & 49.9 & 181.6 & 12.1 & 40210 & 1.64103 \\
\hline$(9)$ & 41.7 & 230.3 & 5.86 & 58910 & 1.66297 \\
\hline$(10)$ & 45.5 & 166.7 & 3.14 & 45200 & 2.01312 \\
\hline$(11)$ & 25.0 & 137.4 & 12.68 & 101100 & 0.87329 \\
\hline
\end{tabular}

(f) $(\mathrm{G}+14) \mathrm{St}$.

\begin{tabular}{|c|c|c|c|c|c|}
\hline $\begin{array}{c}\text { Model } \\
\text { No. }\end{array}$ & $\begin{array}{c}(\mathbf{(}) \\
\mathbf{m m}\end{array}$ & $\begin{array}{c}(\mathbf{(}) \\
\mathbf{m m}\end{array}$ & $\begin{array}{c}(\mathbf{T}) \\
\mathbf{s e c}\end{array}$ & $\begin{array}{c}(\mathbf{V}) \\
\mathrm{kN}\end{array}$ & $\begin{array}{c}\left(\mathbf{T}_{\mathbf{n}}\right) \\
\mathbf{s e c}\end{array}$ \\
\hline$(1)$ & 42.0 & 167.4 & 6.04 & 23210 & 1.94739 \\
\hline$(2)$ & 40.3 & 198.4 & 4.54 & 44260 & 1.27333 \\
\hline$(3)$ & 18.2 & 124.2 & 2.68 & 62660 & 0.87046 \\
\hline$(4)$ & 23.9 & 132.7 & 12.66 & 72530 & 0.77205 \\
\hline$(5)$ & 13.1 & 114.6 & 2.22 & 90860 & 0.68548 \\
\hline$(6)$ & 13.4 & 115.5 & 2.24 & 86830 & 0.71088 \\
\hline$(7)$ & 16.0 & 122.1 & 5.22 & 103200 & 0.64030 \\
\hline$(8)$ & 28.2 & 230.5 & 4.70 & 73300 & 0.99394 \\
\hline$(9)$ & 33.2 & 154.3 & 12.56 & 79820 & 1.02937 \\
\hline$(10)$ & 23.3 & 140.4 & 2.76 & 69480 & 1.30195 \\
\hline$(11)$ & 13.1 & 84.1 & 5.06 & 123300 & 0.57432 \\
\hline
\end{tabular}

(h) $(\mathrm{G}+18) \mathrm{St}$.

\begin{tabular}{|c|c|c|c|c|c|}
\hline $\begin{array}{c}\text { Model } \\
\text { No. }\end{array}$ & $\begin{array}{c}(\mathbf{\Delta}) \\
\mathbf{m m}\end{array}$ & $\begin{array}{c}(\mathbf{(}) \\
\mathbf{m m}\end{array}$ & $\begin{array}{c}(\mathbf{T}) \\
\mathbf{s e c}\end{array}$ & $\begin{array}{c}(\mathbf{V}) \\
\mathrm{kN}\end{array}$ & $\begin{array}{c}\left(\mathbf{T}_{\mathbf{n}}\right) \\
\text { sec }\end{array}$ \\
\hline$(1)$ & 56.9 & 302.7 & 12.14 & 26600 & 2.46286 \\
\hline$(2)$ & 54.2 & 197.2 & 8.40 & 40020 & 1.72411 \\
\hline$(3)$ & 29.4 & 215.5 & 4.78 & 90000 & 1.13759 \\
\hline$(4)$ & 27.7 & 218.2 & 4.72 & 91910 & 1.01590 \\
\hline$(5)$ & 22.1 & 137.7 & 2.72 & 77210 & 0.90770 \\
\hline$(6)$ & 25.8 & 172.4 & 5.76 & 96100 & 0.93863 \\
\hline$(7)$ & 19.7 & 131.3 & 2.68 & 84660 & 0.85189 \\
\hline$(8)$ & 45.4 & 166.9 & 3.14 & 43210 & 1.41704 \\
\hline$(9)$ & 33.8 & 239.6 & 4.82 & 78730 & 1.44451 \\
\hline$(10)$ & 41.9 & 204.7 & 5.80 & 56520 & 1.77149 \\
\hline$(11)$ & 14.0 & 120.9 & 2.24 & 112700 & 0.76884 \\
\hline
\end{tabular}

(j) $(\mathrm{G}+30) \mathrm{St}$.

\begin{tabular}{|c|c|c|c|c|c|}
\hline $\begin{array}{c}\text { Model } \\
\text { No. }\end{array}$ & $\begin{array}{c}(\mathbf{(}) \\
\mathbf{m m}\end{array}$ & $\begin{array}{c}(\boldsymbol{\delta}) \\
\mathbf{m m}\end{array}$ & $\begin{array}{c}(\mathbf{T}) \\
\text { sec }\end{array}$ & $\begin{array}{c}(\mathbf{V}) \\
\mathbf{k N}\end{array}$ & $\begin{array}{c}\left(\mathbf{T}_{\mathbf{n}}\right) \\
\text { sec }\end{array}$ \\
\hline$(1)$ & 98.5 & 416.6 & 5.06 & 23530 & 3.94957 \\
\hline$(2)$ & 192.2 & 556.7 & 11.70 & 51290 & 3.12698 \\
\hline$(3)$ & 67.1 & 263.9 & 8.92 & 57100 & 2.01652 \\
\hline$(4)$ & 50.8 & 237.4 & 8.72 & 55790 & 1.83670 \\
\hline$(5)$ & 39.2 & 172.0 & 6.04 & 58120 & 1.67336 \\
\hline$(6)$ & 51.6 & 189.1 & 6.06 & 57340 & 1.72033 \\
\hline$(7)$ & 49.4 & 161.0 & 14.64 & 61870 & 1.59411 \\
\hline$(8)$ & 78.9 & 521.3 & 5.70 & 60790 & 2.78156 \\
\hline$(9)$ & 100.1 & 332.4 & 12.26 & 57070 & 2.78953 \\
\hline$(10)$ & 77.6 & 429.1 & 6.54 & 60800 & 3.20130 \\
\hline$(11)$ & 54.5 & 230.0 & 5.96 & 89360 & 1.44105 \\
\hline
\end{tabular}

(k) $(\mathrm{G}+40) \mathrm{St}$.

\begin{tabular}{|c|c|c|c|c|c|}
\hline $\begin{array}{c}\text { Model } \\
\text { No. }\end{array}$ & $\begin{array}{c}(\boldsymbol{\Delta}) \\
\mathbf{m m}\end{array}$ & $\begin{array}{c}(\boldsymbol{\delta}) \\
\mathbf{m m}\end{array}$ & $\begin{array}{c}(\mathbf{T}) \\
\mathbf{s e c}\end{array}$ & $\begin{array}{c}(\mathbf{V}) \\
\mathrm{kN}\end{array}$ & $\begin{array}{c}\left(\mathbf{T}_{\mathbf{n}}\right) \\
\mathbf{s e c}\end{array}$ \\
\hline$(1)$ & 68.3 & 261.0 & 3.88 & 16720 & 5.04417 \\
\hline$(2)$ & 67.5 & 353.5 & 5.22 & 31710 & 4.18244 \\
\hline$(3)$ & 91.6 & 463.4 & 5.66 & 57000 & 2.74885 \\
\hline$(4)$ & 79.1 & 411.0 & 6.74 & 64300 & 2.52853 \\
\hline$(5)$ & 98.0 & 326.7 & 6.50 & 76370 & 2.32821 \\
\hline$(6)$ & 96.5 & 355.1 & 6.52 & 79600 & 2.38238 \\
\hline$(7)$ & 66.2 & 346.1 & 12.16 & 74030 & 2.23194 \\
\hline$(8)$ & 89.4 & 422.4 & 4.92 & 52180 & 3.86148 \\
\hline$(9)$ & 171.7 & 568.1 & 11.90 & 66220 & 3.88506 \\
\hline$(10)$ & 205.5 & 490.1 & 14.00 & 74750 & 4.29220 \\
\hline$(11)$ & 58.7 & 277.8 & 8.88 & 88040 & 2.03422 \\
\hline
\end{tabular}


The story displacement for all considered buildings are presented in Fig. (4).

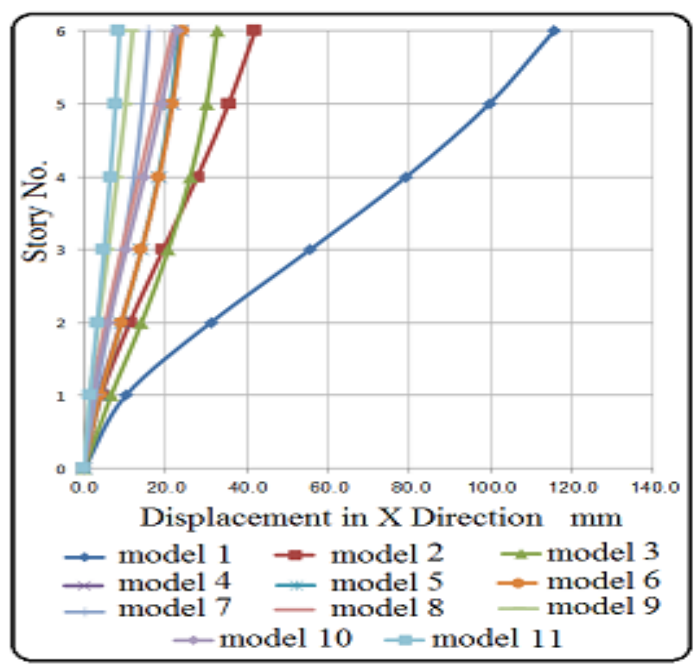

(a) $(\mathrm{G}+4) \mathrm{St}$.

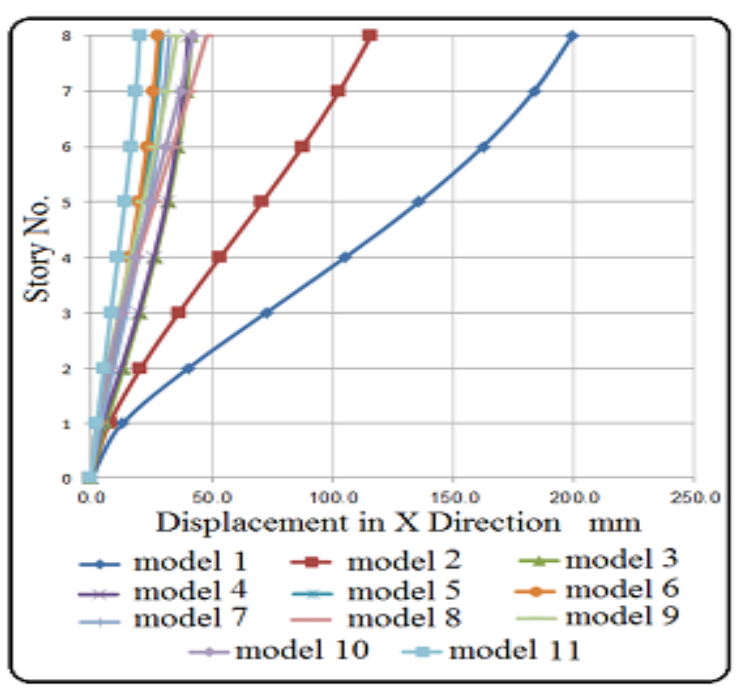

(c) $(\mathrm{G}+8) \mathrm{St}$.

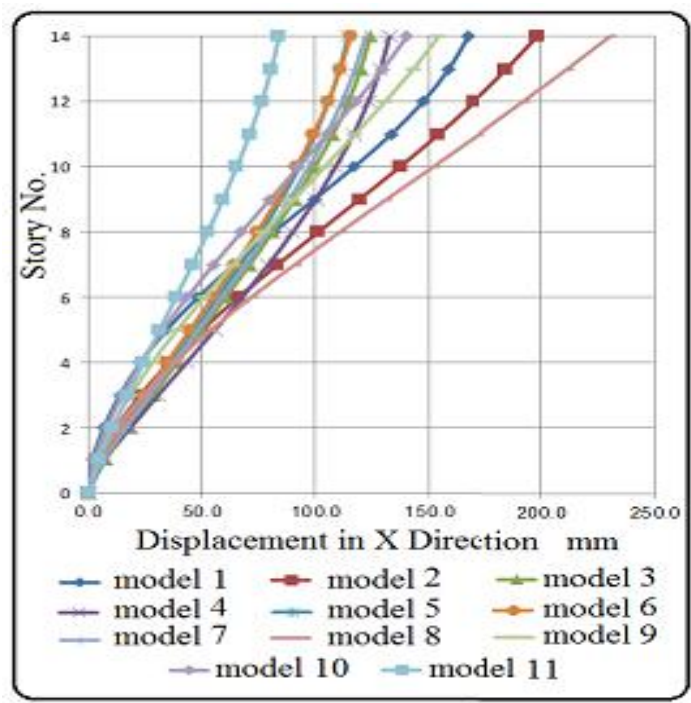

(e) $(\mathrm{G}+12) \mathrm{St}$.

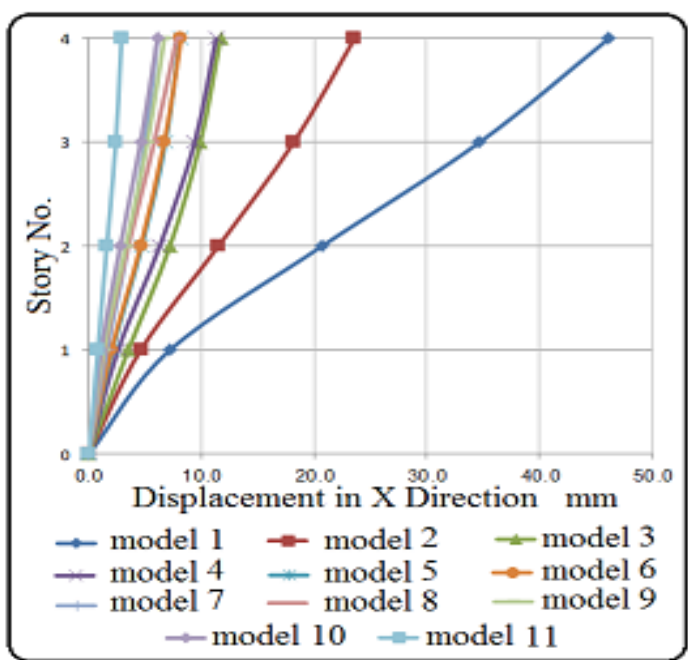

(b) $(\mathrm{G}+6) \mathrm{St}$.

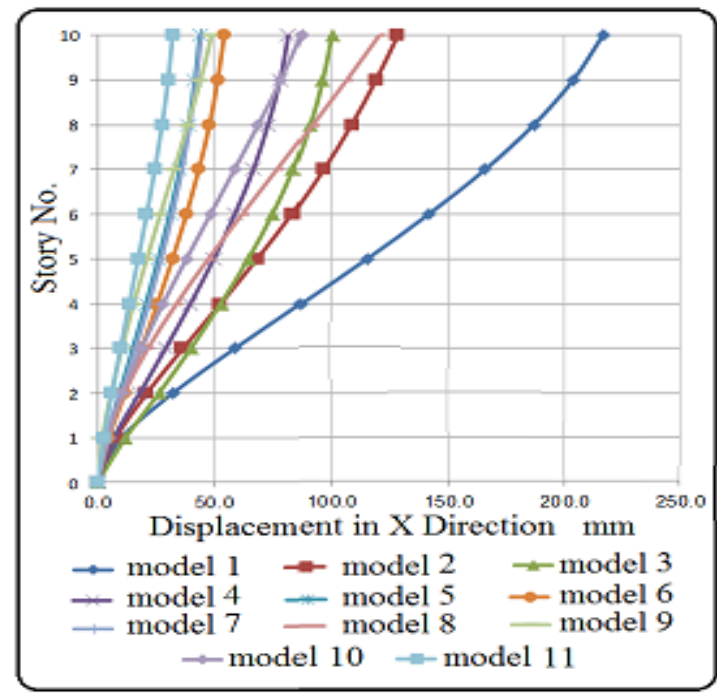

(d) $(\mathrm{G}+10) \mathrm{St}$.

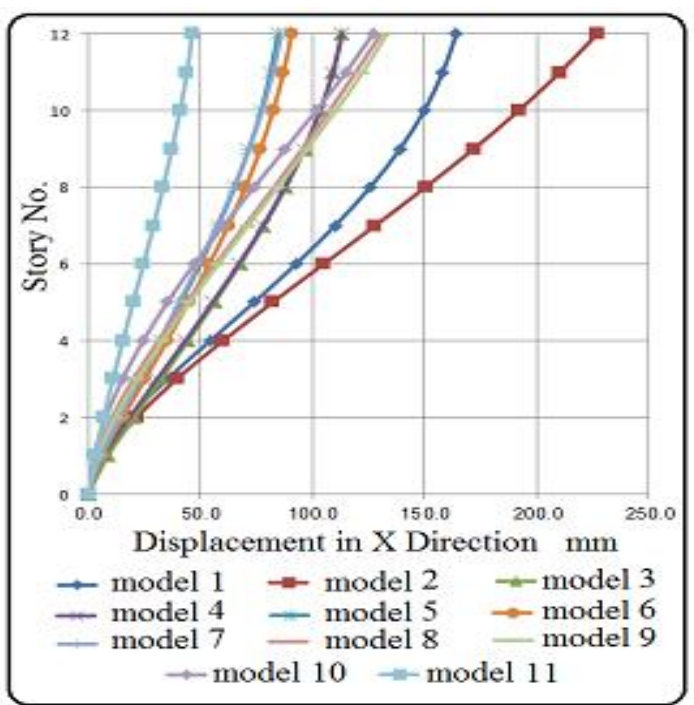

(f) $(\mathrm{G}+14) \mathrm{St}$.

Figure (4) Story displacement for all models 


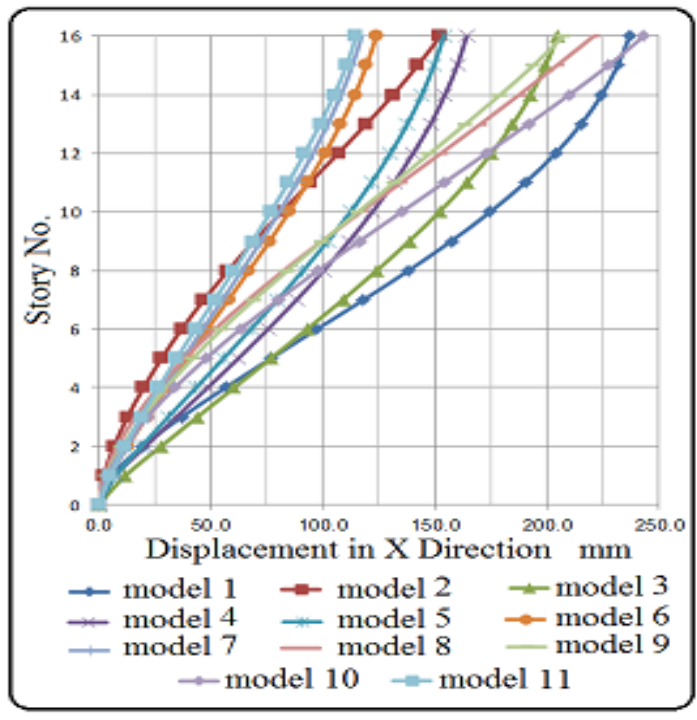

(g) $(\mathrm{G}+16) \mathrm{St}$.

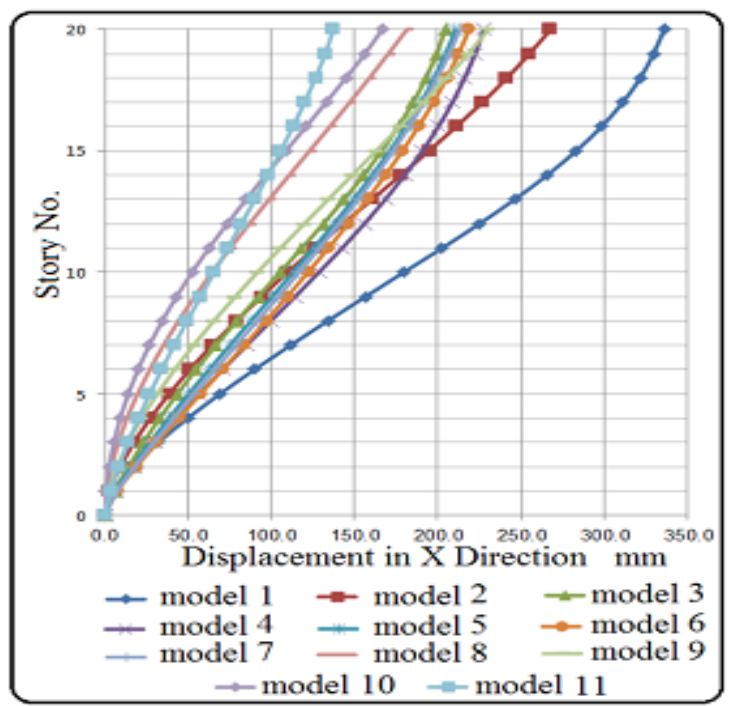

(i) $(\mathrm{G}+20) \mathrm{St}$.

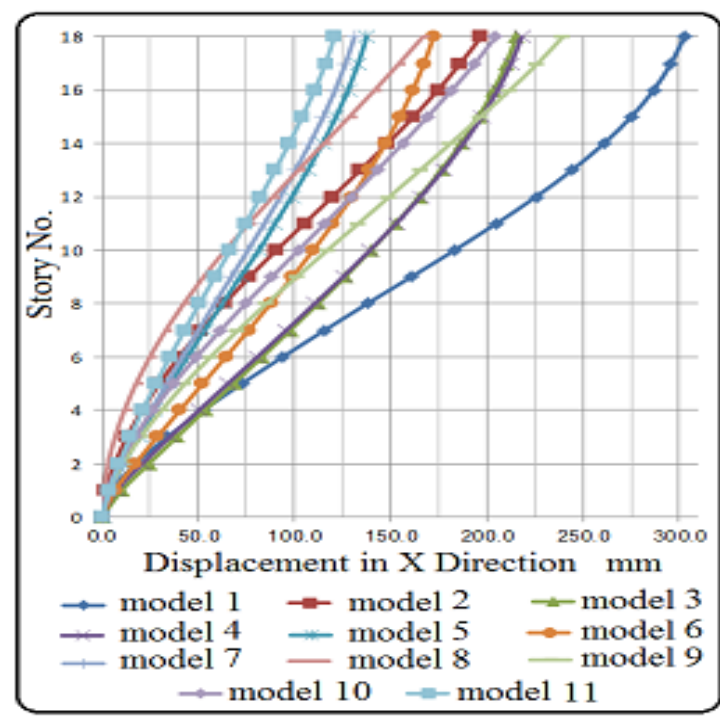

(h) $(\mathrm{G}+18) \mathrm{St}$.

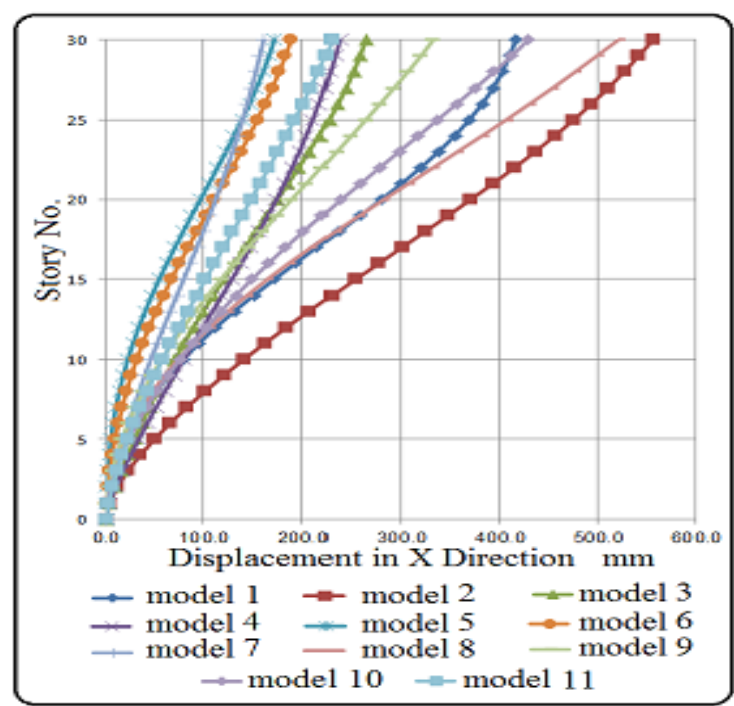

(j) $(\mathrm{G}+30)$ St.

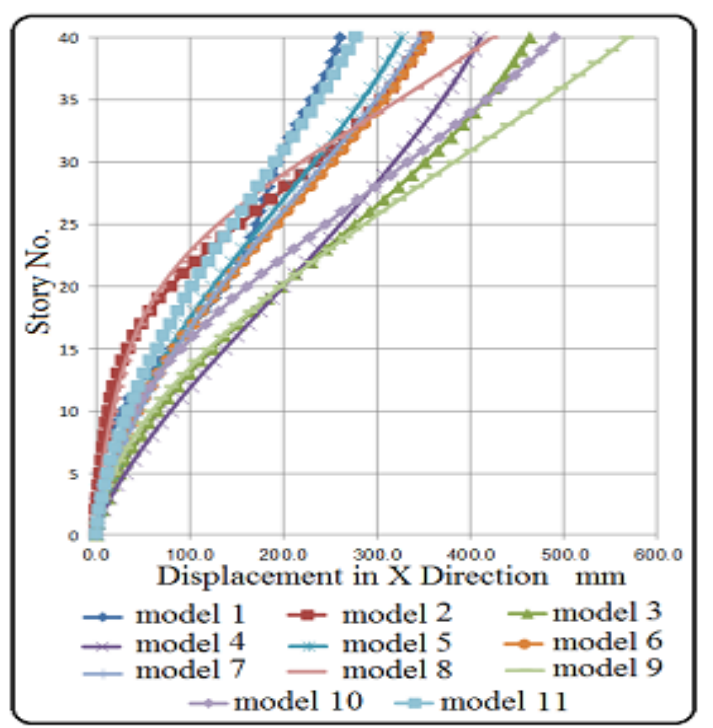

(k) $(\mathrm{G}+40) \mathrm{St}$.

Figure (4) Continued. 


\section{Static Analysis}

The stiffness of $(\mathrm{G}+10,12,14$ and 40$)$ stories buildings are investigated for all types of stiffening systems by applying a $60000 \mathrm{kN}$ lateral static point load in the $\mathrm{X}$ direction at the roof level. These heights of buildings are particularly considered, since for these number of stories the behavior of some studied models reveals special characteristics.This load is proposed as it represents approximately the average value of the base shear in the different studied buildings. The objective of this analysis is to have an assessment of the stiffness of the studied models in order to interpret the stiffness effect on the response of the buildings in the cases of free vibration and earthquake loading. Depending on the definition of stiffness, the stiffer model is the one which gives less displacement. The results are shown in Table (8) and Figs. (5) and (6).

Table (8) Roof displacement for different models of $(\mathrm{G}+10,12,14$ and 40$)$ stories buildings subjected to lateral static point load in $\mathrm{X}$ direction

\begin{tabular}{|c|c|c|c|c|}
\hline \multirow{2}{*}{$\begin{array}{c}\text { Model } \\
\text { No. }\end{array}$} & \multicolumn{5}{|c|}{ Roof displacement (mm) } \\
\cline { 2 - 5 } & $\begin{array}{c}\text { 10 St. } \\
\text { building }\end{array}$ & $\begin{array}{c}\text { 12 St. } \\
\text { building }\end{array}$ & $\begin{array}{c}\text { 14 St. } \\
\text { building }\end{array}$ & $\begin{array}{c}40 \text { St. } \\
\text { building }\end{array}$ \\
\hline 1 & 523 & 631 & 757 & 2530 \\
\hline 2 & 296 & 382 & 483 & 2081 \\
\hline 3 & 120 & 148 & 180 & 793 \\
\hline 4 & 108 & 134 & 163 & 748 \\
\hline 5 & 76 & 96 & 118 & 614 \\
\hline 6 & 80 & 101 & 124 & 638 \\
\hline 7 & 65 & 82 & 102 & 564 \\
\hline 8 & 172 & 243 & 327 & 1754 \\
\hline 9 & 93 & 134 & 185 & 1318 \\
\hline 10 & 129 & 183 & 247 & 1453 \\
\hline 11 & 40 & 53 & 67 & 426 \\
\hline
\end{tabular}

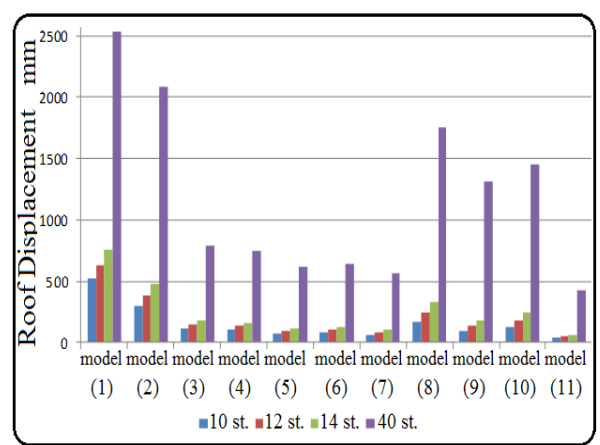

Figure(5) Roof displacement for different modelsof $(\mathrm{G}+10,12,14$ and 40 )stories buildings subjectedto lateral static point load in $\mathrm{X}$ direction

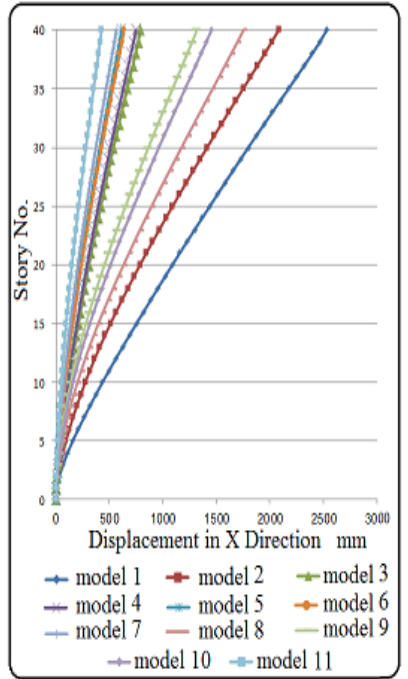

Figure (6) Story displacement for different models of $(\mathrm{G}+40)$ stories buildings subjected to lateral static point load in $\mathrm{X}$ direction 


\section{Free Vibration Analysis}

\section{Discussion of Results}

The fundamental natural period $\left(T_{n}\right)$ of all studied models for all buildings is presented in Fig. (7). The relationships between the height of buildings versus the fundamental natural period for each considered stiffening model are presented in Fig.(8). It appears from Figs. (7) and (8) that the natural time period of a structure is highly affected by its height and properties of the used stiffening system. The fundamental natural period of a building increases by increasing the height of the building. Also,it can be seen that for the same height of a building, the used stiffening system highly affects the fundamental natural period. The fundamental natural period for the building without stiffening is more than other models and it reduces to largest extent for mode(11) in all considered cases. The values of $T_{n}$ for models (1) and (11), from Table(7), are $0.6018 \mathrm{sec}$ and $0.16208 \mathrm{sec}$ for building $(\mathrm{G}+4) \mathrm{st}$, are $1.69764 \mathrm{sec}$ and $0.48463 \mathrm{sec}$ for building $(\mathrm{G}+12)$ st, $2.72803 \mathrm{sec}$ and $0.87329 \mathrm{sec}$ for building $(\mathrm{G}+20) \mathrm{st}$ and $5.04417 \mathrm{sec}$ and $2.03422 \mathrm{sec}$ for building $(\mathrm{G}+$ 40) st.

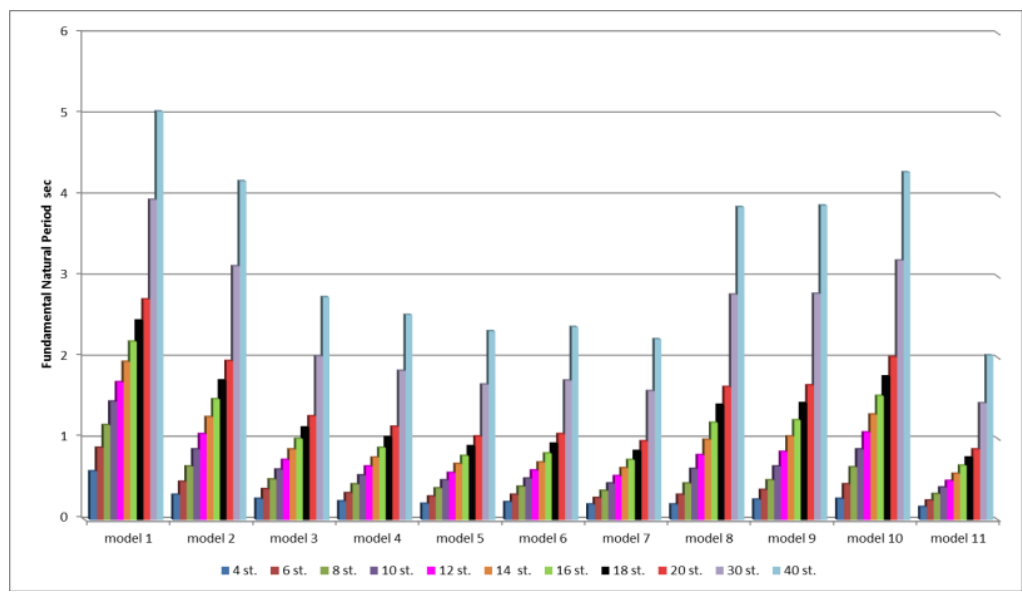

Figure (7) Fundamental natural period $\mathrm{T}_{1}(\mathrm{sec})$ of all studied models and buildings.

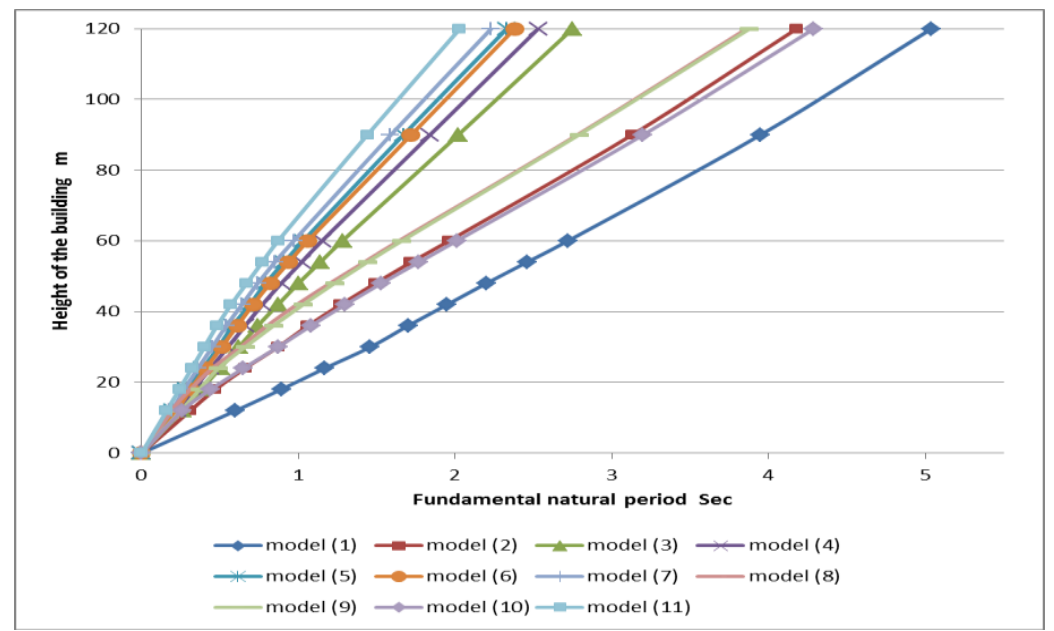

Figure (8)The relationship between the height of buildings versus the fundamental natural period

\section{Response Due to Earthquake Force \\ 2.1 Roof Displacement}

The dynamic analysis results of first four buildings $(G+4,6,8$ and 10) stories show that the maximum lateral roof displacement $(\delta)$ under the action of earthquake force is maximum for the building without stiffening. The results indicate that when the stiffness of a building increases as shown in Fig.(4) and Table (7) the stiffened building undergoes less displacement. The displacement ratio of the maximum roof displacements for the stiffened models to the results of first model (without stiffening), are presented in Table (9).The stiffening model which shows the lesser maximum roof displacement $(\delta)$ under the action of earthquake force for the considered four building is given in Table $(10)$. In this comparisonthe models $\left(2^{\text {nd }}\right.$ to $\left.7^{\text {th }}\right)$ in which bracing is used are treated alone and the models $\left(8^{\text {th }}\right.$ to $\left.10^{\text {th }}\right)$ in which the stiffening is by shear walls is considered alone. However, it should be remembered here that model No.(11) of mixed bracing and shear walls gives least $(\delta)$ displacement. 
Table(9) The displacement ratio of themaximum roof displacements for the buildings of $(G+4,6,8$ and 10$)$ stories

\begin{tabular}{|c|c|c|c|c|}
\hline \multirow{2}{*}{$\begin{array}{c}\text { Model } \\
\text { No. }\end{array}$} & \multicolumn{4}{|c|}{ Displacement ratio } \\
\cline { 2 - 5 } & $\begin{array}{c}\text { 4 St. } \\
\text { building }\end{array}$ & $\begin{array}{c}\text { 6 St. } \\
\text { building }\end{array}$ & $\begin{array}{c}\text { 8 St. } \\
\text { building }\end{array}$ & $\begin{array}{c}10 \mathrm{St} . \\
\text { building }\end{array}$ \\
\hline 1 & 1.00 & 1.00 & 1.00 & 1.00 \\
\hline 2 & 0.51 & 0.36 & 0.58 & 0.59 \\
\hline 3 & 0.25 & 0.28 & 0.21 & 0.46 \\
\hline 4 & 0.24 & 0.21 & 0.20 & 0.38 \\
\hline 5 & 0.18 & 0.20 & 0.15 & 0.20 \\
\hline 6 & 0.18 & 0.21 & 0.14 & 0.25 \\
\hline 7 & 0.13 & 0.14 & 0.16 & 0.21 \\
\hline 8 & 0.17 & 0.19 & 0.24 & 0.56 \\
\hline 9 & 0.15 & 0.10 & 0.18 & 0.23 \\
\hline 10 & 0.13 & 0.20 & 0.21 & 0.40 \\
\hline 11 & 0.06 & 0.07 & 0.10 & 0.15 \\
\hline
\end{tabular}

Table(10) Better braced frame and frame stiffened by shear wall for $(G+4,6,8$ and 10$)$ story buildings under earthquake load

\begin{tabular}{|c|c|c|c|c|}
\hline \multirow{2}{*}{$\begin{array}{c}\text { No. of } \\
\text { stories }\end{array}$} & \multicolumn{2}{|c|}{$\begin{array}{c}\text { Frames stiffened } \\
\text { by bracing } \\
\text { (secondto } \\
\text { seventh models) }\end{array}$} & \multicolumn{2}{|c|}{$\begin{array}{c}\text { Frames stiffened } \\
\text { by shear wall } \\
\text { (eighthto } \\
\text { tenth models) }\end{array}$} \\
\cline { 2 - 5 } & $\begin{array}{c}\text { Better } \\
\text { model }\end{array}$ & $\begin{array}{c}\text { Dis. } \\
\text { ratio }\end{array}$ & $\begin{array}{c}\text { Better } \\
\text { model }\end{array}$ & $\begin{array}{c}\text { Dis. } \\
\text { ratio }\end{array}$ \\
\hline (G+4) & $\begin{array}{c}\text { Model } \\
(7)\end{array}$ & 0.13 & $\begin{array}{c}\text { Model } \\
(10)\end{array}$ & 0.13 \\
\hline$(\mathrm{G}+6)$ & $\begin{array}{c}\text { Model } \\
(7)\end{array}$ & 0.14 & $\begin{array}{c}\text { Model } \\
(9)\end{array}$ & 0.10 \\
\hline$(\mathrm{G}+8)$ & $\begin{array}{c}\text { Model } \\
(6)\end{array}$ & 0.14 & $\begin{array}{c}\text { Model } \\
(9)\end{array}$ & 0.18 \\
\hline \multirow{2}{*}{$(\mathrm{G}+10)$} & $\begin{array}{c}\text { Model } \\
(5)\end{array}$ & 0.20 & $\begin{array}{c}\text { Model } \\
(9)\end{array}$ & 0.23 \\
\hline
\end{tabular}

The results of $(G+12)$ story building shows that the stiffening model 2, which consists of rigid steel frames with $\mathrm{X}$ bracing at corners, gives larger displacement than the one without stiffening (model 1) and all the other models. From the results of the static analysis as shown in Fig.(5) and Table (8) it is found that model 2 is stiffer than model 1 . However, in the dynamic analysis it is observed that the maximum roof displacement of model (2) is more than that of model (1), as shown in Fig.(9). The full time roof response for models (1) and (2) are investigated in Fig.(10). The results show that the roof displacement of model (2) is more than of model (1) only in few seconds of time period of earthquake. Therefore the average roof displacement is proposed as a comparison parameter to have more realistic vision about the behavior of buildings under earthquake force. The results of average roof displacement, Table (7-e), show that during the full time of the applied earthquake load, model (1) which has less stiffness gives more displacement than all other models.
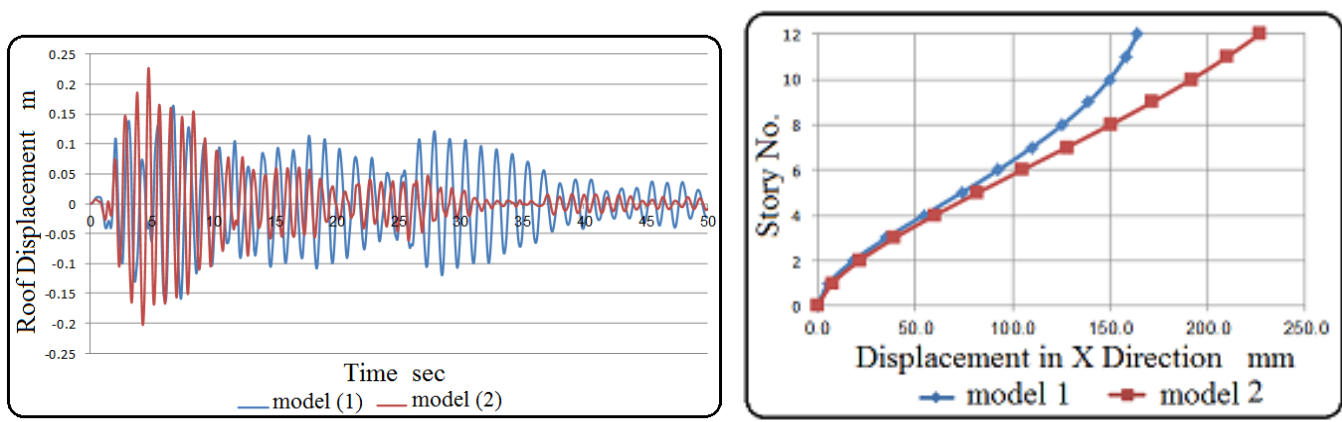

Figure (9) Story displacement for models (1) and (2) of 12 story building

Figure (10) Roof response during full earthquake time for models (1) and (2) of 12 story building 
The same finding that the stiffened building gives larger maximum roof displacement than model (1), which is unstiffened, is also recorded in other buildings of $(\mathrm{G}+14,16$ and 30) stories. Model (2) and (8) of $(\mathrm{G}+14)$ building, model $(10)$ of building $(\mathrm{G}+16)$ and model $(2),(8)$ and $(10)$ of building $(\mathrm{G}+30)$ show this characteristic. This is demonstrated in Figs. (11), (12) and (13). However depending on average roof displacement, it is found that during the full time of the applied earthquake load, the unstiffened model gives more displacement than all other models.

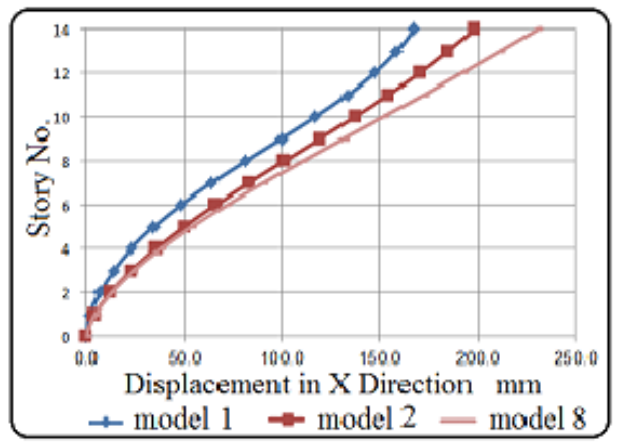

Figure (11) Story displacement for models (1), (2) and (8) of 14 story building

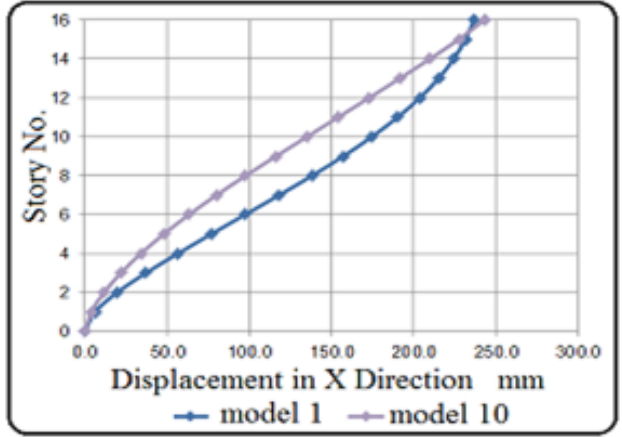

Figure (12) Story displacement for models (1) and (10) of 16 story building

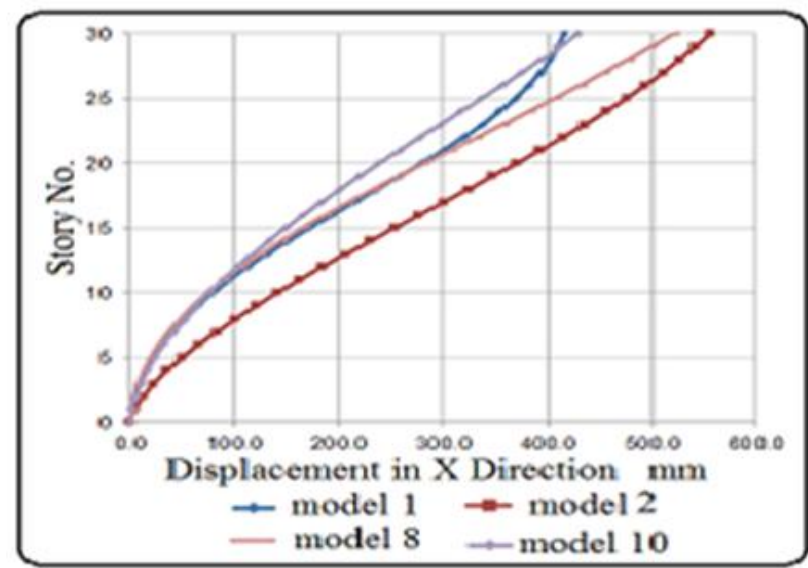

Figure (13) Story displacement for models (1), (2), (8) and (10) of 30 story building

From these results it may be concluded that the roof displacement doesn't give a clear indication on the behavior of the building. Therefore, the full response of the building must be considered. Worthwhile it is to note that for building $(\mathrm{G}+30)$ story the average roof displacement for stiffened models $(2)$ and $(8)$ is larger than that of unstiffened model (1). This can be seen in Table (7-j). The story drifts versus number of stories relationships for model (1) and (10) of $(\mathrm{G}+16)$ story building are shown in Fig. (14). From this Figure it is found that the story drift for model (1) is more than that for model (10) until the ninth story, after which model (10) give larger story drift.

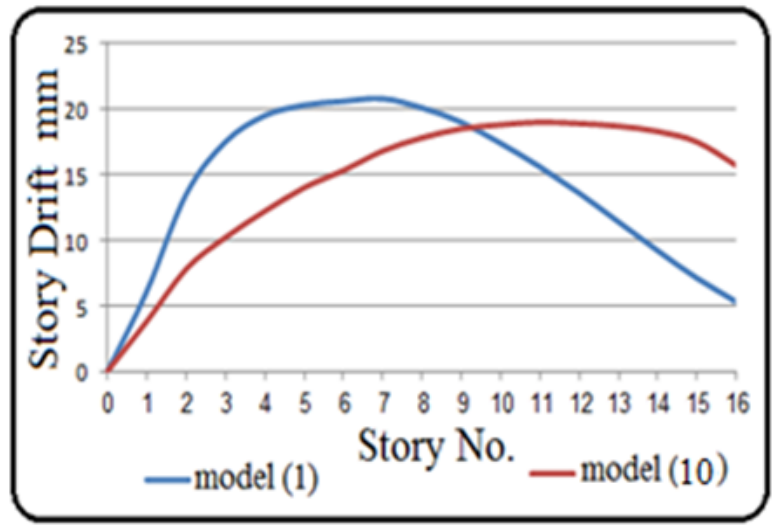

Figure (14) Story drift for models (1) and (10) of 16 story building 
The results of $(G+40)$ story buildings show different behavior relating to the roof displacement. From Table (7-k) the average roof displacement of unstiffened model (1) has smaller value than other models in most cases, during the earthquake time. This leads to the conclusion that under earthquake forces it is not necessarily when the stiffness of a building increases, the roof and story displacement of the building decrease. Theincrease in the building displacement after stiffeningmay be attributedto that these buildings will have a shorter period of vibration, because of its greater stiffness. The shorter period may lead the natural frequency of the structure to approach the applied earthquake vibration and subsequently increase the displacements.

\subsection{Base shear}

The base shear results for the considered heights of buildings show that the effect of the used stiffening system becomes more pronounced in taller structures. The effect of used stiffening system begins to be pronounced in the case of $(\mathrm{G}+10)$ story building. Table $(7)$ shows the results of the base shear $(\mathrm{V})$ for the different buildings. The table reveals that using of bracing and shear walls enhances the base shear.

The base shearratios (R) calculated relative to the value of first model for all buildings are presented in Table (11).

Table (11) Base shear ratio(R)for all buildings

\begin{tabular}{|c|c|c|c|c|c|c|c|c|c|c|c|}
\hline \multirow{2}{*}{$\begin{array}{c}\text { Building } \\
\begin{array}{c}\text { Model } \\
\text { No. }\end{array}\end{array}$} & $\begin{array}{c}\text { St. } \\
\text { St }\end{array}$ & $\begin{array}{c}(\mathrm{G}+6) \\
\text { St. }\end{array}$ & $\begin{array}{c}(\mathrm{G}+8) \\
\text { St. }\end{array}$ & $\begin{array}{c}(\mathrm{G}+10) \\
\text { St. }\end{array}$ & $\begin{array}{c}(\mathrm{G}+12) \\
\text { St. }\end{array}$ & $\begin{array}{c}(\mathrm{G}+14) \\
\text { St. }\end{array}$ & $\begin{array}{c}(\mathrm{G}+16) \\
\text { St. }\end{array}$ & $\begin{array}{c}(\mathrm{G}+18) \\
\text { St. }\end{array}$ & $\begin{array}{c}(\mathrm{G}+20) \\
\text { St. }\end{array}$ & $\begin{array}{c}(\mathrm{G}+30) \\
\text { St. }\end{array}$ & $\begin{array}{c}(\mathrm{G}+40) \\
\text { St. }\end{array}$ \\
\hline$(1)$ & 1.00 & 1.00 & 1.00 & 1.00 & 1.00 & 1.00 & 1.00 & 1.00 & 1.00 & 1.00 & 1.00 \\
\hline$(2)$ & 1.25 & 0.94 & 1.24 & 1.38 & 1.87 & 1.91 & 1.35 & 1.50 & 0.97 & 2.18 & 1.90 \\
\hline$(3)$ & 0.94 & 1.26 & 1.00 & 2.27 & 2.34 & 2.70 & 3.42 & 3.38 & 1.43 & 2.43 & 3.41 \\
\hline$(4)$ & 1.05 & 1.03 & 1.17 & 1.98 & 2.64 & 3.12 & 3.22 & 3.46 & 1.87 & 2.37 & 3.85 \\
\hline$(5)$ & 1.09 & 1.46 & 1.04 & 1.55 & 2.77 & 3.91 & 3.81 & 2.90 & 2.39 & 2.47 & 4.57 \\
\hline$(6)$ & 1.04 & 1.41 & 0.92 & 1.82 & 2.92 & 3.74 & 2.86 & 3.61 & 2.61 & 2.44 & 4.76 \\
\hline$(7)$ & 0.95 & 1.17 & 1.41 & 1.89 & 3.15 & 4.45 & 3.27 & 3.18 & 2.98 & 2.63 & 4.43 \\
\hline$(8)$ & 1.04 & 0.96 & 1.08 & 1.90 & 2.22 & 3.16 & 2.04 & 1.62 & 1.04 & 2.58 & 3.12 \\
\hline$(9)$ & 1.24 & 0.91 & 1.33 & 1.42 & 3.56 & 3.44 & 3.09 & 2.96 & 1.52 & 2.43 & 3.96 \\
\hline$(10)$ & 0.96 & 1.32 & 1.14 & 1.93 & 2.16 & 2.99 & 2.97 & 2.12 & 1.17 & 2.58 & 4.47 \\
\hline$(11)$ & 0.86 & 1.14 & 1.48 & 2.01 & 2.69 & 5.31 & 5.06 & 4.24 & 2.61 & 3.80 & 5.27 \\
\hline
\end{tabular}

\section{VII.Conclusion}

The analysis of the structures with different number of stories and different types of stiffening systems shows that the natural period of a structure is highly affected by the height of the structure and the characteristics and properties of the used improving system. It is directly proportional to its number of stories and inversely proportional, but non-linearly varying with the stiffness of structure. It is found that the stiffness of the structures always increases and is highly affected by using bracing and shear walls. The frame response under earthquake load is highly affected by bracing patterns and shear walls positions. It is found that the base shear is not sensitive in low rise buildings, its effect is obvious in buildings which contain more than ten stories and in most cases the use of different bracing patterns and shear walls enhances the base shear. Also it observed that theroof displacement doesn't give a clear indication on the behavior of the building. The full time response of the building must be considered.The study shows that it is not necessarily when the stiffness of a building increases, the roof and story displacement of the building decrease under earthquake load.

\section{References}

[1] Elnashai, A. S. and Sarno, L. D., Fundamentals of Earthquake Engineering, John Wiley \& Sons, Ltd, United Kingdom, ISBN:978-0-470-02483-6 (Hbk), 1st edition, (2008)

[2] Taranath, B. S., Wind and Earthquake Resistant Buildings, Structural Analysis and Design, Marcel Dekker, ISBN:0-8247-59346, (2005)

[3] Khalaf, M. A., "Simulation of Iraqi Earthquakes and Response of Framed Buildings Using Time and Frequency Domain Analyses". Ph.D. Thesis, the College of Engineering of the University of Al-Basrah, September (2014)

[4] Pundkar, R. S. and Alandkar, P. M., " Influence of Steel Plate Shear Wall on Multistorey Steel Building ", International Journal of Engineering Research and Applications (IJERA), Vol. 3, No. 4, pp. 1940-1945, (2013)

[5] Indian Standard Code, IS 1893-1 (2002): Criteria for Earthquake Resistant Design of Structures, Part 1: General Provisions and Buildings

[6] Verma, S. K.; Roshan, L. and Raman, K., "Seismic response of RC framed buildings ", International Journal of Civil and Structural Engineering, Vol. 4, No. 4, pp. 499-506, (2014)

[7] Dhiman, S.; Nauman, M. and Islam, N., “ Behaviour of Multistory Steel Structure with Different Types of Bracing Systems (A Software Approach) ", International Refereed Journal of Engineering and Science (IRJES), Vol. 4, No. 1, pp. 70-82, (2015)

[8] Chadhar, S. K. and Sharma, A., " Comparative Study of RC Moment Resisting Frame of Variable Heights with Steel Bracing and Shear Wall ", International Journal of Civil and Structural Engineering Research, Vol. 3, No. 1, pp. 220-221, (2015)

[9] Hussain Imran, K. M. and Sowjanya, G. V., " Stability Analysis of Rigid Steel Frames With and Without Bracing Systems under the Effect of Seismic and Wind Loads", International Journal of Civil and Structural Engineering Research, Vol. 2, No. 1, pp. 137-142, (2014)

[10] Indian Standard Code, IS 800 (2007): General Construction In Steel - Code of Practice 
[11] Indian Standard Code, IS 875-1 (1987): Code of Practice For Design Loads (Other Than Earthquake)For Buildings And Structures, Part 1: Dead Loads - Unit Weights of Building Material And Stored Materials

[12] Indian Standard Code, IS 875-2 (1987): Code of Practice for Design Loads (Other Than Earthquake) For Buildings And Structures, Part 2: Imposed Loads

[13] Ross, C.T.F, Finite Element Programs for Structural Vibrations, Springer-Verlag, 1st edition, ISBN-13: 978-1-4471-1888-6, (1991)

[14] CSI Analysis Reference Manual, for SAP2000, ETABS, SAFE andCSi Bridge, Computers \& Structures, Inc., 1978 (2015) 